\title{
ALTERAÇÕES PULMONARES CINTILOGRÁFICAS E ESPIROMÉTRICAS EM PACIENTES JOVENS PORTADORES DE ESQUISTOSSOMOSE MANSÔNICA TRATADOS CLÍNICA E CIRURGICAMENTE NA INFÂNCIA
}

\author{
SCINTIGRAPHIC AND SPIROMETRIC LUNG CHANGES IN YOUNG PATIENTS \\ WITH SCHISTOSOMIASIS MANSONI CLINICALLY AND SURGICALLY \\ TREATED IN CHILDHOOD
}

\author{
Oyama Arruda Frei Caneca ${ }^{1}$ \\ Carlos Teixeira Brandt, TCBC-PE ${ }^{2}$ \\ Waldemar Ladosky ${ }^{3}$ \\ Ricardo Almeida ${ }^{4}$
}

\begin{abstract}
RESUMO: Objetivos: O presente estudo teve por objetivo analisar as alterações pulmonares de pacientes portadores de esquistossomose hepatoesplênica, tratados clínica e cirurgicamente, através da cintilografia pulmonar de perfusão e espirometria. Método: Trinta pacientes jovens portadores de esquistossomose mansônica hepatoesplênica, previamente tratados clínica e cirurgicamente, se submeteram a cintilografia pulmonar de perfusão e avaliação da capacidade respiratória pela espirometria. Resultados: Ao exame cintilográfico, constataram-se alterações em 33,3\% dos pacientes e padrão restritivo pulmonar em $30 \%$ das espirometrias. Conclusões: A prevalência do acometimento pulmonar, avaliado pela Medicina Nuclear, se situou abaixo do observado em pacientes não tratados, relatados na literatura. O padrão restritivo pulmonar, entretanto, avaliado pela espirometria, foi elevado e merece atenção especial no seguimento e manuseio desses pacientes.
\end{abstract}

Descritores: Esquistossomose mansônica; Esquistossomose pulmonar; Cintilografia pulmonar; Espirometria.

\section{INTRODUÇÃO}

O Schistosoma mansoni produz, no sistema venoso portal e no sistema arterial pulmonar, lesões que vão repercutir na hemodinâmica dos referidos sistemas ${ }^{1,2}$. Na fase crônica da doença, observa-se, em cerca de $20 \%$ dos pacientes, além da evolução para a forma hepatoesplênica, o comprometimento pulmonar, aumentando a sua morbidade ${ }^{3-7}$. Os ovos carreados para a circulação arterial pulmonar ficam impactados nas arteríolas de menor diâmetro, determinando a formação de lesões granulomatosas arteriais, parenquimatosas e alveolares que podem levar ao aumento do regime pressórico, determinando hipertensão arterial pulmonar (HAP) ${ }^{8-10}$.
A esquistossomose pulmonar, com ou sem hipertensão arterial pulmonar, pode ser confirmada através dos exames radiológicos do tórax, eletrocardiograma, fonomecanocardiograma, ecodopplercardiograma, estudo hemodinâmico por cateterismo cardíaco e biopsia pulmonar, cada qual com maior ou menor sensibilidade, especificidade e morbidade ${ }^{11}$.

A aplicação das técnicas de medicina nuclear, estudando a forma e a função do órgão, vem evoluindo ultimamente, destacando-se a cintilografia por técnica tomográfica. No caso do pulmão, suas características permitem dois tipos de abordagens: análise da perfusão pulmonar (sistema vascular) e da ventilação pulmonar (sistema traqueobrônquico) ${ }^{12}$.

1. Professor Adjunto de Clínica Pediátrica Cirúrgica da UFPE

2. Professor Titular de Clínica Pediátrica Cirúrgica da UFPE

3. Professor Adjunto de Pneumologia da UFPE

4. Médico do Serviço de Medicina Nuclear da UFPE.

Recebido em 27/06/2001

Aceito para publicação em 09/06/2002

Trabalho realizado no Serviço de Cirurgia Geral da Criança do Hospital das Clínicas da UFPE. 
A espirometria permite avaliar o comprometimento da função respiratória, se obstrutiva (vias áreas) ou restritiva (parênquima pulmonar). O grau de restrição pulmonar, uma vez afastadas outras causas, dá uma idéia da fibrose do parênquima pulmonar, evolução dos granulomas formados pela implantação dos ovos do parasito no interstício do pulmão ${ }^{13}$.

A presente investigação teve por objetivo avaliar o comprometimento pulmonar em 30 pacientes jovens portadores de esquistossomose mansônica hepatoesplênica, previamente tratados clínica e cirurgicamente, quando crianças, através da cintilografia pulmonar de perfusão e da espirometria.

O presente estudo faz parte de uma linha de pesquisa em esquistossomose cirúrgica, devidamente aprovada pelo Comitê de Ética do Hospital das Clínicas da Universidade Federal de Pernambuco.

\section{MÉTODO}

Foram estudados 30 pacientes jovens portadores de esquistossomose mansônica hepatoesplênica associada a varizes sangrentas do esôfago, previamente tratados, quando crianças, clínica e cirurgicamente, segundo protocolo estabelecido no Serviço de Cirurgia Geral da Criança do Hospital das Clínicas da UFPE.

Os pacientes foram selecionados, de forma aleatória, entre os 77 submetidos à esplenectomia, ligadura da veia gástrica esquerda e auto-implante de tecido esplênico no omento maior, de 1990 a 2000, obedecendo o retorno periódico semestral. As idades variaram, no início do tratamento, entre sete e 16 anos. Atualmente, os pacientes têm entre dez e 22 anos (18,0 $\pm 3,1$ anos). Foram analisados 13 pacientes do sexo feminino (43\%) e 17 do sexo masculino $(57 \%)$. Os pacientes estudados apresentavam tempo de seguimento pós-operatório entre 14 a 117 meses $(60,6 \pm 29,2$ meses $)$.

Todos os pacientes se submeteram a ecodopplercardiografia cujos resultados foram considerados dentro de parâmetros normais.

\section{Avaliação Cintilográfica}

Os exames cintilográficos foram realizados utilizando-se uma gama-câmara computadorizada Star Cam 3.000 (GE). O radiofármaco empregado foi um macroagregado de albumina humana (MAA), marcado pelo tecnécio $99 \mathrm{~m}$ TC, obtido no gerador a partir do molibdênio.

Foram adquiridas imagens da distribuição do radiofármaco no ápice e base, face anterior e posterior de ambos os pulmões. Através do computador, determinou-se a contagem média de radioatividade do ápice e base de cada pulmão.

\section{Avaliação da Capacidade Respiratória}

Os exames espirométricos foram realizados em um espirômetro fluxométrico marca Beatrice da EBEM. As medidas do volume de ar exalado e do tempo de expira- ção, além dos parâmetros clássicos da espirometria, foram feitas mediante a utilização do software pulmosoft 4,0y. O cálculo do valor teórico foi feito segundo as normas da SBPT e da CECA ${ }^{13}$.

\section{RESULTADOS}

\section{Cintilografia Pulmonar de Perfusão}

Dos pacientes examinados, $20(66,7 \%)$ apresentaram distribuição homogênea em ambos os pulmões, sem inversão do padrão vascular. Dez $(33,3 \%)$ apresentaram alterações sugestivas de algum comprometimento pulmonar (Figuras 1 e 2).

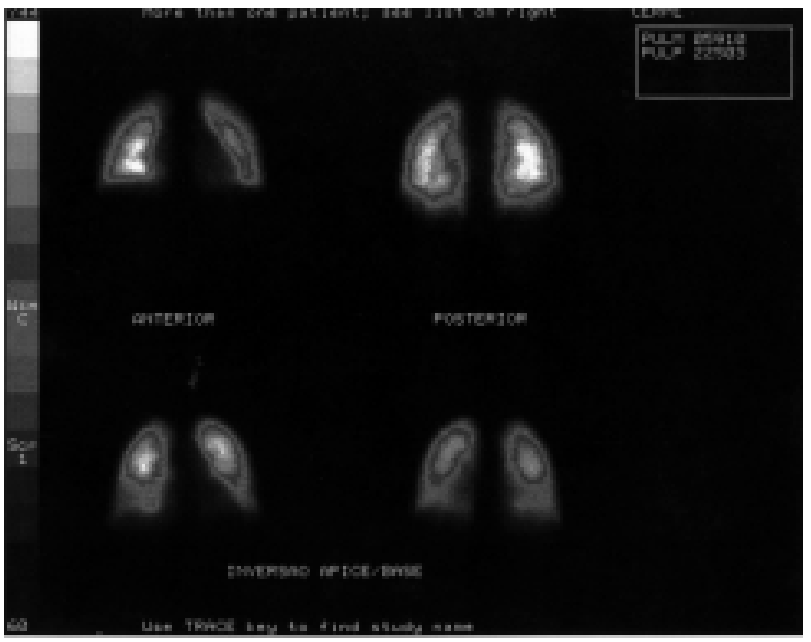

Figura 1 - Cintilografia pulmonar de perfusão. Observa-se inversão do padrão vascular pulmonar, sugerindo hipertensão arterial.

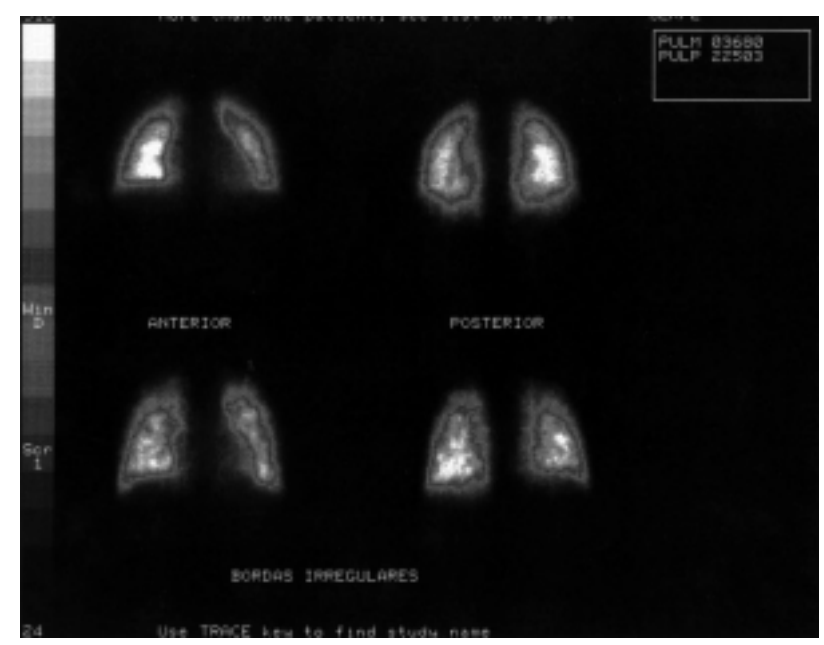

Figura 2 - Cintilografia pulmonar de perfusão. Observam-se limites irregulares com bordas serrilhadas dos pulmões, sugerindo hipertensão arterial. 


\section{Avaliação da Capacidade Respiratória}

Dos espirogramas realizados, 21 (70\%) foram considerados normais, enquanto nove (30\%) apresentaram padrão restritivo pulmonar.

\section{Subgrupos com e SEM AlteraÇões na Cintilografia Pulmonar}

O estudo analítico dos parâmetros respiratórios nos pacientes com e sem alterações cintilográficas pulmonares está exposto nas Tabelas 1 e 2.

Tabela 1

Média e desvio padrão da capacidade vital forçada dos pacientes sem e com alterações na cintilografia pulmonar

\begin{tabular}{l|l}
\hline Sem alteração cintilográfica & Com alteração cintilográfica \\
\hline Média $=88,5 \pm 12,0 \%$ & Média $=84,7 \pm 17,2 \%$ \\
$\mathrm{t}=0,549$ & $\mathrm{p}>0,05$ \\
\hline
\end{tabular}

Tabela 2

Média e desvio padrão do volume expiratório forçado de 1 segundo nos pacientes sem e com alterações na cintilografia pulmonar

\begin{tabular}{l|l}
\hline Sem alteração cintilográfica & Com alteração cintilográfica \\
\hline Média $=94,8 \pm 12,5 \%$ & Média $=90,1 \pm 15,5 \%$ \\
$\mathrm{t}=0,723$ & $\mathrm{p}>0,05$ \\
\hline
\end{tabular}

\section{DISCUSSÃO}

O reconhecimento da hipertensão portopulmonar em portadores de doenças hepáticas graves, candidatos ao transplante de fígado, assim como das repercussões hemodinâmicas no tratamento cirúrgico desses pacientes, vem ensejando cuidados especiais. A utilização da ecodopplercardiografia, na avaliação pulmonar desses pacientes, tem sido a regra, e aceita como um método acurado ${ }^{14-16}$. Entretanto, nos exames ecodopplercardiográficos dos pacientes do presente estudo não foi possível observar alterações significativas que caracterizassem hipertensão pulmonar.

Dos 30 pacientes que realizaram satisfatoriamente a cintilografia pulmonar de perfusão, dez $(33,3 \%)$ apresentaram alterações, sendo três $(10 \%)$ com inversão do padrão vascular, que, uma vez afastada outra doença, permite admitir a presença de aumento da pressão arterial pulmonar. Esses achados são de difícil comparação com os registrados na literatura. Isso se deve ao fato de que na única investigação, utilizando medicina nuclear, descrita na literatura, o radiofármaco usado na propedêutica pulmonar de pacientes esquistossomóticos foi o citrato de
Gálio 67, que avalia a extensão e atividade inflamatória de várias doenças parenquimatosas pulmonares. Nesse trabalho foi verificado mapeamento normal em 43,6\% e hipercaptação em 56,4\% desses pacientes. Embora a investigação por cintilografia pulmonar de perfusão, utilizada no presente estudo, seja diversa do estudo em comparação, os resultados da presente investigação revelam freqüências de acometimento pulmonar inferiores aos encontrados no mesmo ${ }^{6}$. Tal diferença poderia ser explicada, provavelmente, pelas sensibilidades desiguais dos métodos propedêuticos utilizados nos diferentes estudos, assim como, nos contrastes dos padrões dos pacientes examinados, já que os pacientes do estudo de Boueri não tinham recebido tratamento clínico.

Dos espirogramas realizados nos pacientes selecionados, 21 foram normais e nove apresentaram padrão restritivo pulmonar (30\%). Os pacientes do presente estudo não apresentaram ascite ou qualquer doença pulmonar ou abdominal que justificasse um padrão respiratório restritivo. Isto permite admitir que as alterações observadas nos pacientes do presente estudo se devam a lesões parenquimatosas produzidas pelo Schistosoma mansoni. Boueri encontrou padrão restritivo parcial em cinco dos seus 39 pacientes $(12,8 \%)^{6}$

Observou-se que os pacientes com alterações cintilográficas pulmonares apresentaram certa tendência ao padrão respiratório restritivo, apesar das médias da capacidade vital forçada (CVF) e do volume expiratório forçado em um segundo (VEF1) não serem estatisticamente diferentes nos dois subgrupos.

Constatou-se pela análise da pequena casuística do presente estudo comparada com a de outros autores, que houve baixa freqüência do comprometimento pulmonar pelo Schistosoma mansoni. Os pacientes do presente estudo são jovens com idades que variaram de dez a 22 anos, tratados clínica e cirurgicamente quando tinham entre 7 e 16 anos, apresentando atualmente um seguimento médio de cinco anos.

Vários autores admitem a involução espontânea dos granulomas pulmonares esquistossomóticos em pacientes jovens quando não reinfectados, assim como a involução total ou parcial dos granulomas pulmonares após tratamento específico precoce ${ }^{3,5-7,17,18}$. Admite-se, ademais, que, além do tratamento clínico específico, a esplenectomia beneficiaria alguns pacientes com hipertensão pulmonar, diminuindo os níveis pressóricos ${ }^{5,7}$.

A análise do estudo atual, apesar do pequeno número de pacientes, permite inferir que os tratamentos clínico e cirúrgico precoces da esquistossomose mansônica hepatoesplênica nesses jovens podem ter produzido a involução do comprometimento pulmonar pelo parasito, reduzindo a prevalência esperada do acometimento pulmonar. Por outro lado, faz-se necessário uma vigilância maior nos pacientes portadores de esquistossomose cirúrgica com hipertensão pulmonar, particularmente aqueles que apresentam padrão restritivo pulmonar. Tem sido recomendado o uso de prostaciclina, à semelhança de portadores de hipertensão portopulmonar devido a cirrose, nos candidatos ao transplante de fígado. Todavia, essa recomendação ainda requer evidências mais bem fundamentadas ${ }^{19}$. 


\begin{abstract}
Background: The purpose of this investigation was to study with lung scintigraphy and spirometry, the lung involvement in patients with hepatosplenic masonic schistosomiasis previously treated clinically and surgically. Method: Thirty young patients with hepatoesplenic mansonic schistosomiasis previously treated clinical and surgically were examined by pulmonary scintigraphy and spirometry. Results: Pulmonary perfusion scintigraphy detected alterations in $33.3 \%$ of the patients and the spirometry demonstrated a restrictive respiratory pattern in $30 \%$ of them. From these results one can observe a small prevalence of the pulmonary schistosomiasis as compared to the literature. The probable explanation could be due to precocious clinical and surgical treatment, which promote the involution of the granuloma, the basic lesion of the pulmonary schistosomiasis. Conclusions: The prevalence of lung involvement, based on Nuclear Medicine study, was lower than non treated patients reported in the literature, however restrictive respiratory pattern remains high and deserves special attention in the follow up and management of these patients.
\end{abstract}

Key Words: Schistosomiasis mansoni; Pulmonary schistosomiasis; Pulmonary scintigraphy; Spirometry.

\title{
REFERÊNCIAS
}

1. Neves J. Esquistossomose mansoni: Clínica da forma aguda ou toxêmica. Rio de Janeiro: Editora Medsi, 1986.

2. Lambertucci JR, Barraviera B. Esquistossomose mansônica: Estudo clínico. J Bras Med 1994; 67: 59-100.

3. Malta J. Esquistossomose pulmonar In: Malta J. Esquistossomose mansônica. Recife: Editora Universitária UFPE, 1994.

4. Barbosa MM, Lamounier JÁ, Lambertucci J. Acometimento cardiopulmonar na esquistossomose. Arq Bras Cardiol 1995; 65: 343-348.

5. Barbosa MM, Lamounier JA, Oliveira EC, Souza MV, Marques DS, Silva AA, Lambertucci JR. Pulmonary hypertension in schistosomiasis mansoni. Trans Roy Soc Trop Med Hyg 1996; 90: 663-665.

6. Boueri FMV. Avaliação pulmonar na esquistossomose mansônica hepatoesplênica: estudo clínico, radiológico, cintilográfico, funcional e laboratorial. São Paulo, 1993 [Tese, Doutorado, Universidade Federal de São Paulo].

7. Coura JR. Esquistossomose pulmonar. Estudo clínico e experimental. Rio de Janeiro: Editora Cultura Médica Ltda., 1979.

8. Marques RJ. Esquistossomose mansônica pulmonar (Estudo Clínico). Recife, 1951 [Tese, Livre Docência, Faculdade de Medicina da Universidade do Recife].

9. Elian AA. Contribuição da biopsia pulmonar ao estudo da forma assintomática da esquistossomose mansoni pulmonar. Belo Horizonte, 1966 [Tese, Doutorado, Universidade Federal de Minas Gerais].

10. Coura JR, Coutinho SG, Moreaes HM, Dias LB, Rodrigues NP, Silva JR. Esquistossomose pulmonar. O Hospital 1963; 63: 31-50.

11. Malta J, Malta FBL, Malta LBL. Hipertensão arterial pulmonar esquistossomótica. Rev Bras Med 1997; 54:897-910

12. Rocha AFG, Pegado M. Cintigrafia pulmonar. In: Bethlem N. Pneumologia, Rio de Janeiro: Editora Ateneu, 1998, 12: pp163-173.

13. Douce FH. Provas de função pulmonar. In: Scanlan GL, Wilkins RL, Stoller JK. Fundamentos da Terapia Respiratória de Egan. São Paulo: Editora Manole, 2000 .
14. Krowka MJ, Plevak DJ, Findlay JY, Rosen CB, Wiesner RH, Krom RAF. Pulmonary hemodynamics and perioperative cardiopulmonary-related mortality in patients with portopulmonary hypertension undergoing liver transplantation. Liver Transpl Surg 2000; 6 .

15. Lamps LW, Carson K, Bradley AL, Pinson CW, Jonhson JE, Coogan AC, Hunter EB, Clavien PA, Washington MK. Pulmonary vascular morphological changes in cirrhotic patients undergoing liver transplantation. Liver Transpl Surg 1999; 5: 57-64.

16. Ramsay MA, Simpson BR, Nguyen AT, Ramsay KJ, East C, Klintmalm GB. Severe pulmonary hipertension in liver transplant candidates. Liver Transpl Surg 1997; 5:494-500.

17. Silva AE. Aspectos clínico-epidemiológico e evolutivo da hipertensão pulmonar esquistossomótica. Brasília, 1984 [Tese, Mestrado, Faculdade de Ciências Médicas da Universidade de Brasília].

18. Vidal MRF, Barbosa AA, Andrade ZA. Experimental pulmonary schistosomiasis: Lack of morphological evidence of modulation in schistosomal pulmonary granulomas. Rev Inst Med Trop São Paulo 1993; 35: 423-429.

19. Krowka MJ, Frantz RP, Mcgoon MD, Severson C, Plevak DJ, Wiesner RH. Improvement in pulmonary hemodynamics during intravenous epoprostenol (prostacyclin): a study of 15 patients with moderate to severe portopulmonary hypertension. Hepatology, 1999; 30:642-648

Endereço para correspondência:

Dr. Carlos Teixeira Brandt

Rua Boa Viagem, 5.090/1201

51030-000 — Recife-PE 